Hier können Sie CME-Punkte sammeln a) für die Pflichtfortbildung aller Vertragsärzte und b) für freiwillige Fortbildungszertifikate, die viele Landesärztekammern anbieten.

Die Multiple-Choice-Fragen beziehen sich auf den vorangegangenen Fortbildungsbeitrag (Seiten 40-43). Die Antworten ergeben sich z. T. aus dem Text, z. T. beruhen sie auf medizinischem Basiswissen!
Dr. H. J. K. Barwitz, Prof. Dr. A. Berghaus, Prof. Dr Dr. h.c. Th. Brandt, Prof. Dr. W. G. Daniel, Prof. Dr. W. Eisenmenger, Prof. Dr. K. Friese, Prof. Dr. H. S. Füessl, Prof. Dr. B. Göke, Prof. Dr. R. Gradinger, Prof. Dr. H. Holzgreve, Prof. Dr. A. Imdahl, Prof. Dr. K.-W. Jauch, Prof. Dr. K. Krüger, Prof. Dr. H.-J. Möller, Prof. Dr. D. Reinhardt, Prof. Dr. Dr. h.c. Th. Ruzicka, Prof. Dr. Ch. Stief, U. Weigeldt.

\title{
Fragen zum Thema „Osteoporosetherapie in der Hausarztpraxis“
}

\section{- 1. Wer ist besonders gefährdet für osteo- porotische Knochenbrüche? \\ A Prämenopausale Frauen \\ B Junge Männer \\ C Alle Frauen nach dem 50. Lebensjahr \\ D Frauen nach dem 70. Lebensjahr \\ E Alle Männer nach dem 50. Lebensjahr}

\section{- 2. Welche Maßnahme hilft, osteoporo-} tische Brüche im Alter zu verringern?

\section{A Rauchen}

B Sportliche Aktivitäten meiden

C Fettarme Diät einhalten

D Kalziumarm ernähren

E Vitamin-D-Versorgung optimieren

\section{- 3. Ab welchem Body-Mass-Index ist das osteoporotische Frakturrisiko deutlich erhöht?}

A $<20$

B $>20$

C $>25$

D $>30$

E Das Gewicht hat keinen Einfluss auf die Bruchgefahr.

\section{- 4. Welche TSH-Werte sind bei den mit Schilddrüsenhormonen behandelten Patienten mit einem zwei- bis vierfach erhöhten Bruchrisiko verbunden?}

\section{A $>0,3 \mathrm{mU} / \mathrm{l}$}

B $>1,0 \mathrm{mU} / \mathrm{l}$

C $0,5-1,0 \mathrm{mU} / \mathrm{l}$

D $<0,3 \mathrm{mU} / \mathrm{l}$

E Der TSH-Wert hat keinen Einfluss auf das Bruchrisiko.

\author{
- 5. Welche(r) Laborparameter sollte(n) in \\ der Praxis zum Ausschluss einer Osteo- \\ malazie bestimmt werden? \\ A Parathormon \\ B Serum-Kalzium und alkalische Phos- \\ phatase \\ C Calcitriol \\ D BSG \\ E Kalzium-Ausscheidung im Urin
}

- 6. Welche der folgenden Personen sollte eine medikamentöse Osteoporosetherapie erhalten?

A 72-jährige Frau mit einem DXA-TWert von $-3,5$

B 40-jährige Frau mit einem DXA-TWert von $-2,5$

C 60-jähriger Mann mit einem DXA-TWert von $-2,5$

D 70-jähriger Mann mit einem Unterarmbruch und einem DXA-T-Wert von $-1,0$

E 80-jährige Frau mit einem DXA-TWert von $-1,5$

- 7. Was gilt es vor Beginn einer medikamentösen Osteoporosetherapie zu beachten?

A Mit einer medikamentösen Therapie sollte man im Frühstadium beginnen.

B Eine mögliche Osteomalazie sollte abgeklärt werden.

C Osteoporosemedikamente verhindern keine peripheren Frakturen.

D Kombinationstherapien sind einer Monotherapie vorzuziehen.

E Wenn bereits Brüche vorliegen, kommt eine medikamentöse Therapie zu spät.

\section{- 8. Was ist die Standardmethode der Knochendichtemessung? \\ A Ultraschall an der Ferse \\ B Quantitatives CT an der Wirbelsäule \\ C DXA \\ D Peripheres quantitatives CT \\ E MRT}

- 9. Bei welcher Konstellation ist eine Therapie mit Bisphosphonaten zu empfehlen?

A 69-jähriger Mann ohne Risikofaktoren mit einem DXA-T-Wert von $-2,8$

B 67-jährige Frau ohne Risikofaktoren mit einem DXA-T-Wert von -2,5

C 79-jährige Frau mit Tumorkachexie bei diffuser Leber- und Lungenmetastasierung eines Kolonkarzinoms

D 72-jährige Frau mit Steroid-Langzeittherapie über drei Monate

E 72-jähriger Mann mit Risikofaktoren und einem DXA-T-Wert von $-1,8$

- 10. Was ist bei der Osteoporosetherapie mit Bisphosphonaten zu beachten?

A Der Therapieerfolg lässt sich gut am Anstieg der Knochendichte überprüfen.

B Ein Abfall der Knochendichte unter der Therapie ist üblich.

C Ein fehlender Anstieg der Knochendichte bedeutet kein Therapieversagen.

D Kontrollen der Knochendichte sollten in jährlichen Abständen erfolgen.

E Kontrollen der Knochendichte sollten in halbjährlichen Abständen erfolgen.

\section{ANTWORTFORMULAR}

Mit Einreichen dieses Fragebogens erkläre ich mich damit einverstanden, dass die angegebenen Daten zum Zweck der Teilnahmebestätigung gespeichert und bei erfolgreicher Teilnahme an den Einheitlichen Informationsverteiler (EIV) der Ärztekammern weitergegeben werden.

Wichtiger Hinweis:

Eine Auswertung ist künftig nur noch möglich, wenn Sie Ihre EFN auf dem Antwortformular angeben! Nähere Hinweise hierzu unter: www.cme-punkt.de/faq.html

\section{"Osteoporosetherapie in der Hausarztpraxis"}

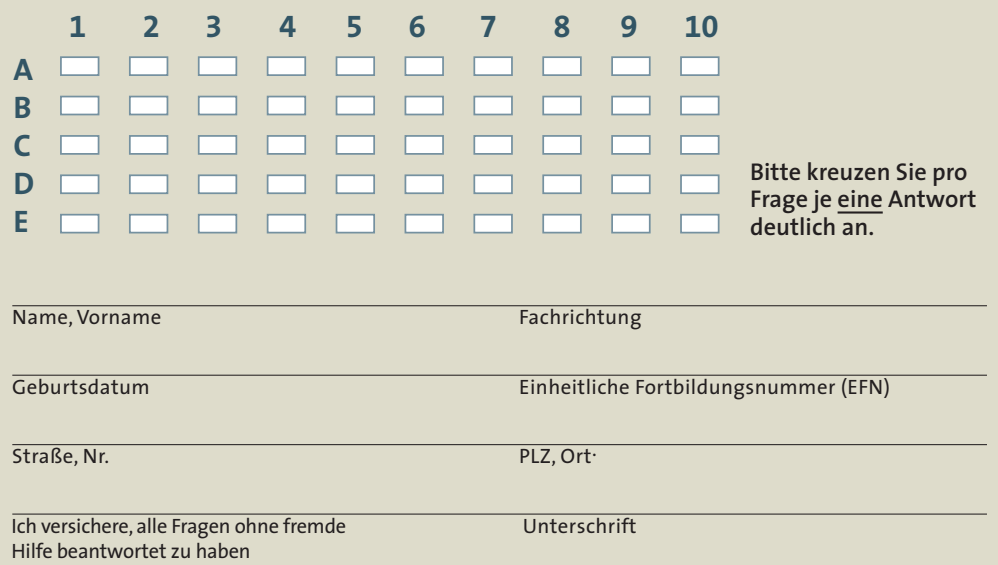

Und so kommen Sie zu Ihren Punkten:

Teilnahme im Internet: unter www.cme-punkt.de. Dort führen wir für Sie ein elektronisches Punktekonto.

Teilnahme per Brief: Fragebogen ausfüllen und mit frankiertem Rückumschlag an: Urban \& Vogel GmbH CME MMW-Fortschr. Med. Postfach 81664 München. 